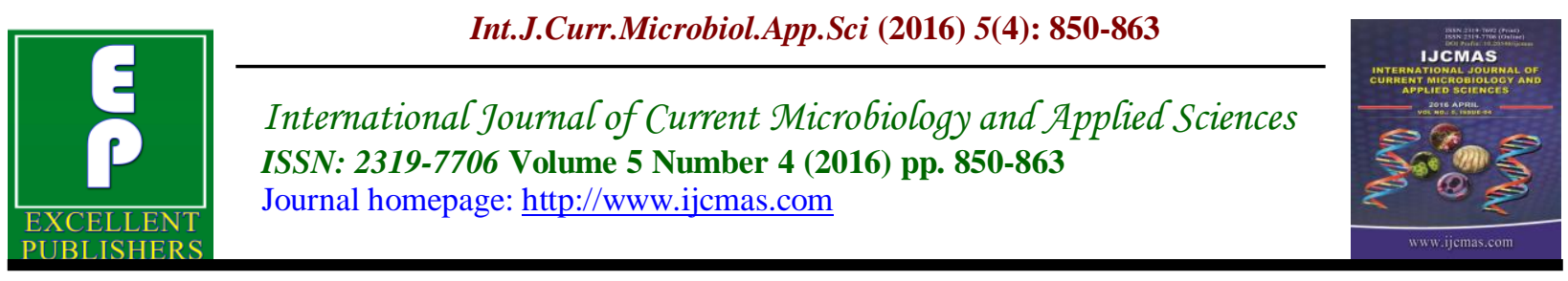

Original Research Article

http://dx.doi.org/10.20546/ijcmas.2016.504.097

\title{
Effect of Intercropping and Phosphorus Fertilizer Treatments on Incidence of Rhizoctonia Root-Rot Disease of Faba Bean
}

\author{
Abeer M. Mousa ${ }^{1}$ and Sahar A. El-Sayed ${ }^{2}$ \\ ${ }^{1}$ Soil and Water Research Department, Nuclear Research Center, Atomic Energy \\ Authority, Cairo, Egypt \\ ${ }^{2}$ Plant Pathology Institution, Agriculture Researcher Center, Egypt \\ *Corresponding author
}

\begin{abstract}
A B S T R A C T
Keywords

Faba bean, Intercropping, phosphorus fertilizer, Rhizoctonia solani and root-rot

\begin{tabular}{l}
\hline Article Info \\
\hline Accepted: \\
25 March 2016 \\
Available Online: \\
10 April 2016
\end{tabular}

In greenhouse and field experiments, conducted in two successive winter seasons 2012/2013\& 2013/2014 the effect of intercropping three cultivars of fababean (Giza3 Mohassan \& Giza 40 and Sakha1) with wheat (Sakha 93). As well as the effect of phosphorus fertilizer treatments on incidence of root rot of three cultivars faba bean, as well as yield component three cultivars faba bean, as well as yield component was studied. Application of phosphorus fertilizer (100 and $200 \mathrm{Kg} / \mathrm{fed}$ ) as superphosphate $\left(15.5 \% \mathrm{P}_{2} \mathrm{O}_{5}\right)$ before planting caused a reduction in incidence and disease severity on plants of the three cultivars of faba bean in greenhouse and field experiments. In field experiments, were intercropping and phosphorus fertilization at (100 and $200 \mathrm{Kg} / \mathrm{fed}$ ) respectively, which reduced the root-rot diseases. Intercropping three cultivars of faba bean (Giza 3 Mohassan Giza 40 and Sakha 1) with wheat Sakha93 and phosphorus fertilizer significantly increased yield characters i.e., plant height, number of branches, number of pods plant, 100 seeds/weight and seed weight $\mathrm{ardad} / \mathrm{fed}$.
\end{abstract}

\section{Introduction}

Faba bean (Vicia faba L.) is used as an important human food in developing countries and as an animal feeds mainly for pigs horses, poultry and pigeons in industrialized countries. Feeding value of faba bean is high and this legume has beanconsiders a meat extender of substitute due to its high protein content (20-41\%) (Chavan et al., 1989). Root-rot and wilt diseases caused by several soil borne fungal pathogens are wide spread and serious in many crop cultivated in different soil types.
Faba bean is subjected to attack by many pathogenic organisms wherever the crop grown. In This respect, root-rot disease is among the most important fungal diseases affecting faba bean production in Egypt. Omer (1986) tested several pathogens, and found that Rhizoctonia solani was the most pathogenic in causing root-rot disease. Metwely (2004) found that Rhizoctonia solani isolated from Kafr El- sheikh governorate was more virulent than that of Sharkia governorate. Several root-rot and 
wilt pathogens such as Rhizoctonia solani, Fusarium solani, Macrophomina phaseolina are reported to attack faba bean roots and stem base causing serious losses in seed germination and plant stand as well (Adelkader et al., 2011). Intercropping can be defined as the growth of two or more crops in the same space at the same time (Andrews and Kassam 1976).

This technology may enable the intensification of a forming system, leading to increased general productivity and biodiversity in the intercropped fields as compared to monocultiveres of the individual intercropped species (Vandermeer et al., 1998). Furthers, intercropping can be advantageous for controlling plant diseases such as common bacterial blight and fungal rust (Boudreau and Mundt 1992, Fininsa 1996). In organic field trials, a disease reduction has bean observed in intercrops of barley (Hordeum vulgare L.) with pea (Pisum sativum L.), faba bean disease reduction has been observed in intercrops of barley (Hordeum vulgare L.) with pea (Pisum sativum L.), faba bean (Vicia faba L.) and lupin (Lupinus sp.) Kinane and Lyngkjaer 2002). In addition increasing diversity through intercropping many reduce pest and disease incidence leading to less pesticide application increased habitat for beneficial insects and microorganisms, and an overall reduction in farm inputs (Kontturi et al., 2011). Development of disease resistant variety was the most economical mean of control. Phosphorus has been called (the key to life) because it is directly involved in most life process. It exerts many and varied functions in plant metabolism and hence inadequate phosphate supply to the plant seriously affect numerous metabolic processes. Singh et al., (1981) found that phosphorus applied at 60 and $90 \mathrm{Kg} / \mathrm{ha}$ significantly increased yields in comparison with $30 \mathrm{Kg} \mathrm{P}$. When four level of $\mathrm{P}(0,30$, 60 and $90 \mathrm{Kg} / \mathrm{ha}$ ) were applied. Negm et al., (1992) reported that phosphorus application increased the number of branches and flowers per plant. Jain et al., (1999) and Khurana and Sharma (2000) showed that the combined inoculation of Rhizobium and phosphate solubilizing bacteria increased nodulation, yield of pea and total biomass of chickpea compared with either individual inoculation or the uninoculated control (Rudresh et al., 2005). Rania et al., (2010) found that the soil application of phosphorus fertilizer increased plant height, number of branches/plant, number of pods/plant, 100 seed weight/g and seed weight ardab/fed in both seasons of the three legume verities. Jafar (2014) found that, application of biological phosphorus fertilizer significantly increased the yield and yield components in faba bean plant.

\section{Materials and Methods}

This work has been combined out at experimental Farm of Sers El-layian Agricultural Research station (SEARS), Minufiya Governorate, Egypt. The mechanical and chemical properties of the experimental soil for the upper foot layer (0$30 \mathrm{~cm}$ ) and available phosphorus of the experimental soil table (1) were determined before sowing in the two seasons according to the method described by (Chapman and Pratt 1961) and presented in table (1). Mechanical and chemical properties of the experimental sites revealed that the texture of the soil was sandy clay with a low $\mathrm{P}$ concentration the two growing seasons table (1)

\section{Source of the Pathogen}

A pathogenic isolate of Rhizoctonia solani was selected from the gene bank of the Plant Pathology Research Institute - Agricultural 
Research Centre. The isolate was tested to confirm its virtence.

\section{Greenhouse Experiments}

\section{Determination the Effect of Intercropping between Three Cultivars Faba Bean and Wheaton Root-rot Incidence}

Three cultivars faba bean C.V. Giza 3 Mohassan, Giza 40 and sakha1 were intercropping with wheat (sakha 93) under greenhouse conditions atSers El-layian Agricultural Research station, in 2012 growing season. Pots $(25 \mathrm{~cm})$ were sterilized by dipping in $5 \%$ formalin solution for 5 min and then left in a pone air till dryness. Soil sterilization was accomplished with 50 $\%$ formalin solution mixed thoroughly, covered with plastic sheet for one week and then the plastic sheet was removed in order to complete formalin evaporation. Soil infestion with each individual fungus $R$. solaniwas carried out at the rate of the rate of $3.5 \%$ of soil weight. Fungus was individually grown on sand-barley (SB) medium (25 clean sand 759 barley and enough water to cover the mixture).

Flasks contained sterilized medium were incubated with each particular fungus and incubated at $25^{\circ} \mathrm{C}$ for two weeks. Potted soil was watered daily for week to enhance fungal growth. Soil of control pots was mixed with the same amount of sterilized sand- barley (SB) medium.

Five surface sterilized seeds of faba bean were sown in the opposite side with the transplanted wheat (Sakha 93) in each pot. While five faba bean seeds were sterilized and only treatment. Percentage of pre- and post-emergence damping -off and survival plant were recorded at 15 and 30 days. While disease severity was estimated at 45 days after sowing (Soleman et al., 1988).

\section{Determination the Effect of Different Rates from Phosphorus Fertilizer Treatments on Root-rot Incidence}

Pots $(25 \mathrm{~cm})$ filled with $5 \mathrm{Kg}$ clay soil were sterilized as mentioned before than the soil was infested with the inocula of the fungus $(R$. solani) at rate of $3.5 \% \mathrm{w} / \mathrm{w}$. as mentioned before. The phosphorus fertilizer rates ( 0,100 and $200 \mathrm{~kg} / \mathrm{fa}$ ) was mixed with the top later of the soil before sowing. Five seeds of fababean (Giza 3 Mohassan, Giza 40 and sakhal were sown in each pot, and three replicates were used for each treatment. Percentages of pre-and postemergence damping-off as well as healthy survival plants in each treatment were determined 15 days after sowing, respectively using the next formula according to El-Helaly et al.,(1970) and disease severity was estimated at 45 days after sowing.

\section{Field Experiments}

Determination the Effect of Intercropping between Three Cultivars of Faba Bean and Wheat (Sakha 3) in Naturally Infested Soil

These experiments were conducted in naturally infested soil at Sers El-layian Agriculture Research Station (SEARS),Munfiya governorate, Egypt, during two winter season (2012/2013). The experimental layout was in completely randomized block design. The plot of $3 \mathrm{~m} \mathrm{x}$ $3.5 \mathrm{~m}$ was used in this study. Four plots contained five rows (apart) per each assembled treatment wheat Shakha93 was individually intercropping with each of the tested three cultivar of faba bean (c.v. Giza 3 Mohassan Giza 40 and sakha 1) were used: 2 row (faba bean with 2 row wheat), 3 row (faba bean with 3 row wheat) and 2 row (faba bean with 4 row wheat). 
Control plot had five rows of faba bean plants only.

The recorded data were:-

Pre-emergence damping-off after 15 days from sowing.

Post-emergence damping-off and survival plants after 30 days from sowing.

Average plant height after harvest, number of branches, number of pods/plant, 100 seeds/weight and total yield/fed were recorded.

\section{Determination of the effect of fertilization phosphorus treatments faba bean in naturally infested soilon root-rot disease}

Two field experiments were carried out in the experimental Farm of SEARS,Munfiya governorate, Egypt, during two winter season (2012/2013) and ( 2013/2014). Each experiment included three cultivars of faba bean (Giza 3 Mohassan Giza 40 and Sakha 1) in the main plots extraction solution of phosphorus fertilizer was prepared by dissolved calcium superphosphate $15.5 \%$ $\left(\mathrm{P}_{2} \mathrm{O}_{5}\right)$. For each experiment, a spilt - split plots design with three replicates of five rows. The plot size was $6 \mathrm{~cm}$ consisting of five rows, each row 3 meter long spaced at $60 \mathrm{~cm}$. the rates of phosphorus fertilization $(0,100$ and $200 \mathrm{~kg} / \mathrm{fa})$ were applied as super phosphate $15.5 \% \mathrm{P}_{2} \mathrm{O}_{5}$ before planting.

The recorded data were:-

Pre-emergence damping-off after 15 days from sowing.

Post-emergence damping-off and survival plants after 30 days from sowing.

Average plant height, fresh weight and dry weight after 45 days from sowing.
At maturity, plants were harvest and the following yield characters i.e. plant height, number of branches, number of pods/plant, 100 seeds/weight and total yield/fed were recorded.

Seed samples of each crops were taken and dried, then ground to powder for chemical analysis. Nitrogen content was determined using improving macro-kjeldahl method (A.O.A.C. 1980). The percentage of seed protein content was calculated by multiplying the percentage of nitrogen content was determined calorimetrically using spectrophotometer according to Chapman and Pratt (1961).

\section{Statistical Analysis}

All collected data were subjected to statistical analysis for each season and to combined analysis over years according to Gomez and Gomez (1984).

\section{Results and Discussion}

Effect of Intercropping between Three Cultivars of Faba Bean with Wheat Plants in Greenhouse Conditions

As shown in table (2) intercropping three faba bean with wheat significantly reduced both pre- and post-emergence damping-off root rot disease caused by the fungal pathogens. Rhizoctonia solani compared to untreated one (control). However all cultivars significantly reduced the disease severity of root-rot symptoms caused by Rhizoctonia solani.

\section{Effect of Super Phosphate on Root-rot Incidence, under Greenhouse Condition}

Data in table (3) clearly show the effect of super phosphate $(0,100,200 \mathrm{Kg} / \mathrm{fed})$ on the percentage of pre- and post- emergence damping-off, root-rot disease severity 
caused by the fungal pathogens, $R$. soloni. All tested phosphorus fertilizer significantly reduced the development of root-rot disease, the highest percentage of survival plant in all three cultivars faba bean was resulted in soil fertilized with super phosphate at 200 $\mathrm{Kg} / \mathrm{fed}$ ).

\section{Effect of Intercropping between Three Cultivars of Faba Bean with Wheat on Root-rot Under Field Conditions}

This experiment was conducted during two successive seasons (2012/2013) and (2013/2014) to study the effect of intercropping between three cultivars of faba bean with wheat on root-rot infestion and some related growth characters. In this respect data in table (4) show that the intercropping in form of row (faba bean X 4 row wheat), of the first season (2012/2013) inciteda significant reduction in preemergence and post-emergence damping off of three cultivars faba bean with wheat respectively.

In the second season similar trend of result was detected with minor variation in the rank. Healthy fababean survival plants were increased in case of three cultivars of faba bean with wheat than the corresponding faba bean with wheat than the corresponding faba bean grown alone.

As for the effect of intercropping between three cultivars of faba bean with wheat on disease incidence, growth and yield component of faba bean under field conditions, the obtained data in table (5) revealed significant positive effects of intercropping when compared with the control where they lowered the disease incidence while increased the plant height, number of branches, number of pods per plant, 100-seed weight and seed weight ardab/fed.
Effect of Phosphorus Fertilizers on the Incidence of Three Cultivars Faba Bean Root- rot under Field Conditions

Data in table (6) clearly show the influence of super phosphate $(0,100$ and $200 \mathrm{~kg} / \mathrm{fed}$.) on pre- and post- emergence damping-off, three cultivars of faba bean under field conditions during two seasons. The results revealed that application of both fertilizer rates (100 and $200 \mathrm{~kg} / \mathrm{fed})$ significantly reduced pre- and post- emergence dampingoff when compared to the untreated plants (control). Both fertilizer rates (100 and 200 $\mathrm{kg} / \mathrm{fed}$.) the highest level of the disease control with the highest number of survived plants. In general fertilizer applied degree offered by superphosphate fertilizer applied at rate of $200 \mathrm{~kg} / \mathrm{fed}$ was much higher then untreated control.

Data in table $(7,8)$ revealed of significant effect of fertilization with super phosphate over the control where these effect were shown in case of plant height, fresh weight, dry weight and plant height after harvest, number of branches, number of pods, 100 seed weight and seed weight $\mathrm{ardab} / \mathrm{fed}$. the obtained results were confirmed from the two experiments conducted in 2012/20132013/2014 seasons respectively. However, the great effect on plant growth and yield component was given by super phosphate at $200 \mathrm{~kg} / \mathrm{fed}$ ) application compared with nonfertilized control.

Effect of Intercropping Three Cultivars of Faba Bean with Wheat and Super Phosphate Fertilizers on Chemical Composition of Faba Bean Plants

The present results indicated that the intercropping three cultivars of faba bean and super phosphate fertilizers with different rates applied to soils (0,100 and $200 \mathrm{~kg} / \mathrm{fed})$ caused significant increase in phosphorus, nitrogen contents in there cultivars faba bean. 
Table.1 Physical and Chemical Properties of the Experimental Soil for Upper Foot Layer (0-30 Cm ) in the Two Growing Seasons

\begin{tabular}{|c|c|c|c|c|c|c|c|c|c|c|c|c|c|c|c|c|c|c|c|c|}
\hline \multirow{3}{*}{$\begin{array}{l}\text { season } \\
\text { s }\end{array}$} & \multirow[t]{3}{*}{ sites } & \multirow{3}{*}{$\begin{array}{l}\text { Textur } \\
\text { e }\end{array}$} & \multicolumn{4}{|c|}{ Physical properties } & \multicolumn{14}{|c|}{ Chemical properties } \\
\hline & & & \multirow[t]{2}{*}{ coarse } & \multirow[t]{2}{*}{ Fine } & \multirow[t]{2}{*}{ silt } & \multirow[t]{2}{*}{ clay } & \multirow{2}{*}{$\begin{array}{l}\mathrm{pH} \\
(1: 2.5 \\
)\end{array}$} & \multirow{2}{*}{$\begin{array}{l}\text { E.C.m } \\
\text { mhas/c } \\
\text { m } \\
20{ }^{\circ} \mathrm{C}\end{array}$} & \multirow{2}{*}{$\begin{array}{l}\mathrm{CaCO}_{3} \\
\%\end{array}$} & \multicolumn{4}{|c|}{ Cations $\mathrm{ml} / 100 \mathrm{~g}$} & \multicolumn{4}{|c|}{ Anions $/ \mathrm{ml} / 100 \mathrm{~g}$} & \multirow[t]{2}{*}{$\mathrm{N} \%$} & \multirow[t]{2}{*}{$\mathrm{P} \%$} & \multirow[t]{2}{*}{$\mathrm{K} \%$} \\
\hline & & & & & & & & & & $\mathrm{Ca}^{++}$ & $\mathrm{Mg}^{++}$ & $\mathrm{Na}^{+}$ & $\mathrm{K}^{+}$ & $\mathrm{Co}_{3}^{--}$ & $\mathrm{HCo}_{3}$ & $\mathrm{Cl}^{-}$ & $\mathrm{So}_{4}^{--}$ & & & \\
\hline $\begin{array}{l}2012 / 2 \\
013\end{array}$ & 1 & $\begin{array}{l}\text { Sandy } \\
\text { clay }\end{array}$ & 1.7 & 51.1 & 25 & 19 & 7.40 & 0.37 & 2.3 & 1.3 & $\begin{array}{l}1.1 \\
0\end{array}$ & 1.14 & 0.43 & --- & 1.0 & 1.3 & 1.90 & 0.12 & 0.11 & 0.02 \\
\hline $\begin{array}{l}2013 / 2 \\
014\end{array}$ & 2 & $\begin{array}{l}\text { Sandy } \\
\text { clay }\end{array}$ & 1.9 & 50.9 & 26 & 20 & 8.10 & 0.38 & 2.5 & 1.6 & $\begin{array}{l}1.2 \\
5\end{array}$ & 1.20 & 0.50 & 0.5 & 1.4 & 1.6 & 1.21 & 0.18 & 0.13 & 0.04 \\
\hline
\end{tabular}

Table.2 Effect of Intercropping Three Cultivars of Faba Bean and Wheat, Grown in Soil Infested with Specific Pathogen Species Of Rhizoctoniasoloni under Greenhouse Conditions

\begin{tabular}{|l|c|c|c|c|}
\hline Cultivars & $\begin{array}{c}\text { Pre- } \\
\text { emergence } \\
\text { damping-off }\end{array}$ & $\begin{array}{c}\text { Post- } \\
\text { emergence } \\
\text { damping-off }\end{array}$ & $\begin{array}{c}\text { Survival } \\
\text { plant }\end{array}$ & $\begin{array}{c}\text { Disease } \\
\text { severity }\end{array}$ \\
\hline Faba bean (Giza 3 Mohassan)+ wheat & 13.33 & 13.33 & 73.34 & 40.00 \\
Faba bean (Giza 40)+ wheat & 16.67 & 18.67 & 64.66 & 53.33 \\
Faba bean (Sakha 1)+ Wheat & 10.0 & 13.33 & 76.67 & 30.00 \\
Faba bean (Giza 3 Mohassan) & 23.33 & 20.0 & 56.67 & 63.33 \\
Faba bean (Giza 40) & 26.62 & 23.33 & 50.05 & 68.88 \\
Faba bean (Sakha 1) & 18.86 & 13.33 & 67.81 & 43.33 \\
\hline L.S.D at 0.05 & 8.21 & 8.34 & 12.83 & 6.91 \\
\hline
\end{tabular}


Table.3 Effect of Superphosphate on Root-rot Incidence, under Greenhouse Condition

\begin{tabular}{|c|c|c|c|c|c|c|}
\hline $\begin{array}{l}\text { Cultivars } \\
\text { of faba } \\
\text { bean }\end{array}$ & $\begin{array}{c}\text { Concentrations of } \\
\text { superphosphate } \\
\text { as kg/fed }\end{array}$ & fungus & $\begin{array}{l}\text { Pre-emergence } \\
\text { damping -off \% }\end{array}$ & $\begin{array}{l}\text { Post-emergence } \\
\text { damping off \% }\end{array}$ & $\begin{array}{l}\text { Survival } \\
\text { plant } \%\end{array}$ & $\begin{array}{c}\text { Disease } \\
\text { severity } \%\end{array}$ \\
\hline \multirow{4}{*}{$\begin{array}{l}\text { Giza } 3 \\
\text { Mohassan }\end{array}$} & 10 & \multirow{4}{*}{ R. solani } & 20.00 & 13.33 & 66.67 & 55.55 \\
\hline & 100 & & 10.00 & 10.0 & 80.0 & 40.00 \\
\hline & 200 & & 6.67 & 3.37 & 89.96 & 29.99 \\
\hline & Mean & & 12.22 & 8.9 & 78.88 & 41.85 \\
\hline \multirow[t]{4}{*}{ Giza 40} & 10 & \multirow[t]{4}{*}{ R. solani } & 23.33 & 16.67 & 60.0 & 67.77 \\
\hline & 100 & & 20.00 & 13.33 & 66.67 & 55.55 \\
\hline & 200 & & 13.33 & 10.00 & 78.67 & 36.66 \\
\hline & Mean & & 18.99 & 13.33 & 67.78 & 53.32 \\
\hline \multirow[t]{4}{*}{ Sakha 1} & 0 & \multirow[t]{5}{*}{ R. solani } & 10.00 & 10.00 & 80.0 & 40.00 \\
\hline & 100 & & 6.67 & 6.67 & 86.66 & 28.88 \\
\hline & 200 & & 3.34 & 3.33 & 93.33 & 20.00 \\
\hline & Mean & & 6.66 & 6.67 & 86.66 & 29.63 \\
\hline \multicolumn{2}{|r|}{ L.S.D at 0.05} & & 7.53 & 7.15 & 11.23 & 5.89 \\
\hline
\end{tabular}

Table.4 Effect of Intercropping Three Cultivars of Faba Bean with Wheat Incidence of Root-

Rot Disease during Two Seasons Under Field Conditions

\begin{tabular}{|c|c|c|c|c|c|c|c|}
\hline \multirow[t]{2}{*}{ cultivars } & \multirow[t]{2}{*}{ Intercropping } & \multicolumn{3}{|c|}{$2012 / 2013$} & \multicolumn{3}{|c|}{$2013 / 2014$} \\
\hline & & $\begin{array}{l}\text { Pre- } \\
\text { emergence } \\
\text { damping - } \\
\text { off \% }\end{array}$ & $\begin{array}{l}\text { Post- } \\
\text { emergence } \\
\text { damping } \\
\text { off \% }\end{array}$ & $\begin{array}{l}\text { Surviv } \\
\text { al plant } \\
\%\end{array}$ & $\begin{array}{l}\text { Pre- } \\
\text { emergence } \\
\text { damping - } \\
\text { off \% }\end{array}$ & $\begin{array}{l}\text { Post- } \\
\text { emergence } \\
\text { damping } \\
\text { off } \%\end{array}$ & $\begin{array}{l}\text { Surviva } \\
1 \text { plant } \\
\%\end{array}$ \\
\hline \multirow{5}{*}{$\begin{array}{l}\text { Giza3 } \\
\text { Mohassan }\end{array}$} & 2 rowX 2 row (wheat) & 0.0 & 6.67 & 93.33 & 3.33 & 6.67 & 90.0 \\
\hline & 3 rowX 3 row (wheat) & 3.33 & 3.33 & 93.34 & 6.67 & 6.67 & 86.66 \\
\hline & 2 rowX 4 row (wheat) & 0.0 & 10.0 & 90.0 & 3.33 & 10.0 & 86.67 \\
\hline & Faba bean alone & 16.67 & 16.67 & 66.66 & 16.67 & 20.00 & 63.33 \\
\hline & mean & 5.00 & 9.17 & 85.83 & 7.5 & 10.84 & 81.67 \\
\hline \multirow{5}{*}{ Giza 40} & 2 rowX 2 row (wheat) & 3.33 & 10.0 & 86.67 & 6.67 & 6.67 & 86.66 \\
\hline & 3 rowX 3 row (wheat) & 10.00 & 10.0 & 80.00 & 6.67 & 10.0 & 83.33 \\
\hline & 2 rowX 4 row (wheat) & 6.67 & 10.0 & 83.33 & 13.33 & 10.0 & 76.67 \\
\hline & Faba bean alone & 20.0 & 16.67 & 63.33 & 20.0 & 13.33 & 66.67 \\
\hline & mean & 10.0 & 11.67 & 78.33 & 11.67 & 10.0 & 78.33 \\
\hline \multirow{8}{*}{ Sakha1 } & 2 rowX 2 row (wheat) & 0.0 & 3.33 & 96.67 & 3.33 & 3.34 & 93.33 \\
\hline & 3 rowX 3 row (wheat) & 3.33 & 0.0 & 96.67 & 3.33 & 6.67 & 90.0 \\
\hline & 2 rowX 4 row (wheat) & 0.0 & 0.0 & 100.0 & 0.0 & 3.33 & 96.67 \\
\hline & Faba bean alone & 10.0 & 20.0 & 70.0 & 13.33 & 10.0 & 76.67 \\
\hline & mean & 3.33 & 5.83 & 90.87 & 4.99 & 5.84 & 89.17 \\
\hline & L.S.D at 0.05 & 3.15 & 2.91 & 6.00 & 4.35 & 4.83 & 8.55 \\
\hline & & 6.11 & 6.18 & 8.91 & 6.73 & 4.00 & 10.23 \\
\hline & & 8.27 & 6.33 & 10.14 & 8.20 & 7.38 & 14.41 \\
\hline
\end{tabular}


Table.5 Effect Intercropping Three Cultivars of Faba Bean with Wheat on Some Yield Component Plant during Two Seasons under Field Conditions

\begin{tabular}{|c|c|c|c|c|c|c|c|c|c|c|c|}
\hline \multirow[t]{2}{*}{ cultivars } & \multirow[t]{2}{*}{ Intercropping } & \multicolumn{5}{|c|}{$2012 / 2013$} & \multicolumn{5}{|c|}{$2013 / 2014$} \\
\hline & & $\begin{array}{l}\text { Plant } \\
\text { height } \\
\text { after } \\
\text { harvest }\end{array}$ & $\begin{array}{l}\text { Branches } \\
\text { plant }\end{array}$ & $\begin{array}{l}\text { No } \\
\text { seeds } \\
\text { pods }\end{array}$ & $\begin{array}{l}100 \\
\text { seed } \\
\text { weight } \\
\mathrm{g}\end{array}$ & $\begin{array}{l}\text { Seed } \\
\text { weight } \\
\text { ardab/fed }\end{array}$ & $\begin{array}{l}\text { Plant } \\
\text { height } \\
\text { after } \\
\text { harvest }\end{array}$ & $\begin{array}{l}\text { Branches } \\
\text { plant }\end{array}$ & $\begin{array}{l}\text { No } \\
\text { seeds } \\
\text { pods }\end{array}$ & $\begin{array}{l}100 \\
\text { seed } \\
\text { weight } \\
\mathrm{g}\end{array}$ & $\begin{array}{l}\text { Seed } \\
\text { weight } \\
\text { ardab/fed }\end{array}$ \\
\hline \multirow{5}{*}{$\begin{array}{l}\text { Giza3 } \\
\text { Mohassan }\end{array}$} & 2 rowX 2 row (wheat) & 100.0 & 3.00 & 19.05 & 83.00 & 3.31 & 103 & 2.90 & 18.0 & 81.0 & 3.00 \\
\hline & 3 rowX 3 row (wheat) & 101.0 & 3.05 & 21.00 & 89.00 & 3.55 & 105 & 3.11 & 20.0 & 85.0 & 3.30 \\
\hline & 2 rowX 4 row (wheat) & 98.0 & 3.95 & 23.00 & 92.00 & 4.00 & 101 & 3.50 & 22.0 & 88.0 & 3.50 \\
\hline & Faba bean alone & 90.1 & 2.99 & 14 & 75.00 & 3.00 & 93 & 2.75 & 12.0 & 73.0 & 2.88 \\
\hline & mean & 97.28 & 3.25 & 19.26 & 84.75 & 3.47 & 100.5 & 3.07 & 18 & 81.75 & 3.17 \\
\hline \multirow{5}{*}{ Giza 40} & 2 rowX 2 row (wheat) & 90.0 & 2.90 & 17.0 & 70.0 & 2.90 & 85.0 & 2.33 & 16.0 & 68.0 & 2.70 \\
\hline & 3 rowX 3 row (wheat) & 93.0 & 3.0 & 19.0 & 72.0 & 2.95 & 89.0 & 2.67 & 18.0 & 70.0 & 2.90 \\
\hline & 2 rowX 4 row (wheat) & 90.0 & 3.0 & 20.0 & 75.0 & 3.00 & 93.0 & 2.77 & 19.0 & 75.0 & 3.15 \\
\hline & Faba bean alone & 85.0 & 2.33 & 12.0 & 60.0 & 2.55 & 81.0 & 2.10 & 11.0 & 58.0 & 2.40 \\
\hline & Mean & 89.5 & 2.81 & 17.00 & 69.25 & 3.09 & 87 & 2.47 & 16.00 & 67.75 & 2.97 \\
\hline \multirow{5}{*}{ Sakha1 } & 2 rowX 2 row (wheat) & 109.0 & 3.55 & 22.0 & 90.0 & 4.30 & 110.0 & 3.14 & 23.0 & 88.0 & 4.00 \\
\hline & 3 rowX 3 row (wheat) & 113.0 & 3.95 & 24.0 & 95.0 & 4.40 & 115.0 & 3.31 & 24.0 & 93.0 & 4.30 \\
\hline & 2 rowX 4 row (wheat) & 108.0 & 4.00 & 26.0 & 98.0 & 4.60 & 112.0 & 3.50 & 25.0 & 95.0 & 4.50 \\
\hline & Faba bean alone & 101.0 & 3.40 & 16.0 & 80.0 & 3.90 & 105.0 & 3.00 & 17.0 & 78.0 & 3.73 \\
\hline & Mean & 107.75 & 3.73 & 22.00 & 90.75 & 4.3 & 110.5 & 3.24 & 22.25 & 88.5 & 4.13 \\
\hline \multirow{3}{*}{\multicolumn{2}{|c|}{ L.S.D. at 0.05}} & 1.08 & 0.15 & 0.43 & 0.32 & 0.61 & 1.22 & 0.11 & 0.47 & 0.35 & 4.13 \\
\hline & & 2.91 & 0.21 & 1.33 & 1.00 & 0.88 & 1.98 & 0.28 & 1.09 & 1.19 & 0.12 \\
\hline & & 3.50 & 0.53 & 1.52 & 1.24 & 1.18 & 3.67 & 0.57 & 1.58 & 1.40 & 0.22 \\
\hline
\end{tabular}


Table.6 Effect of Different Rates (0,100,200 Kg/Fed) of Superphosphate Soil Fertilizer on Incidence of Root-Rot Disease of Cultivars of Faba Bean during Two Seasons under Field Conditions.

\begin{tabular}{|c|c|c|c|c|c|c|c|}
\hline \multirow[t]{3}{*}{ Cultivars } & \multirow{3}{*}{$\begin{array}{l}\text { Concentra } \\
\text { tions of } \\
\text { superphos } \\
\text { phate } \\
\text { as kg/fed }\end{array}$} & \multicolumn{6}{|c|}{ Disease incidence } \\
\hline & & \multicolumn{3}{|c|}{$2012 / 2013$} & \multicolumn{3}{|c|}{$2013 / 2014$} \\
\hline & & $\begin{array}{l}\text { Pre- } \\
\text { emergence } \\
\text { damping - } \\
\text { off } \%\end{array}$ & $\begin{array}{l}\text { Post- } \\
\text { emergence } \\
\text { damping- } \\
\text { off \% }\end{array}$ & $\begin{array}{l}\text { Survival } \\
\text { plant } \%\end{array}$ & $\begin{array}{l}\text { Pre- } \\
\text { emergence } \\
\text { damping - } \\
\text { off } \%\end{array}$ & $\begin{array}{l}\text { Post- } \\
\text { emergenc } \\
\text { e } \\
\text { damping } \\
\text { off \% }\end{array}$ & $\begin{array}{l}\text { Survival } \\
\text { plant } \%\end{array}$ \\
\hline \multirow{4}{*}{$\begin{array}{l}\text { Giza3 } \\
\text { Mohassan }\end{array}$} & 0 & 16.67 & 10.0 & 73.33 & 16.67 & 13.33 & 70.0 \\
\hline & 100 & 6.67 & 3.3 & 90.0 & 10.00 & 13.33 & 76.67 \\
\hline & 200 & 3.34 & 3.33 & 93.33 & 3.34 & 6.67 & 89.99 \\
\hline & Mean & 8.89 & 5.55 & 85.55 & 10.00 & 11.11 & 78.89 \\
\hline \multirow{4}{*}{ Giza 40} & 0 & 20.0 & 16.67 & 63.33 & 23.34 & 18.33 & 58.33 \\
\hline & 100 & 16.67 & 13.33 & 70.0 & 20.00 & 16.67 & 63.33 \\
\hline & 200 & 10.0 & 10.00 & 80.0 & 16.67 & 13.33 & 70.00 \\
\hline & Mean & 15.56 & 13.33 & 71.11 & 20.00 & 16.11 & 63.89 \\
\hline \multirow{4}{*}{ Sakh1 } & 0 & 10.0 & 3.34 & 86.66 & 13.34 & 10.0 & 76.66 \\
\hline & 100 & 0.0 & 3.34 & 96.66 & 6.67 & 3.33 & 90.0 \\
\hline & 200 & 0.0 & 0.00 & 100.0 & 3.34 & 3.33 & 93.33 \\
\hline & Mean & 3.33 & 2.22 & 94.44 & 7.78 & 5.55 & 86.66 \\
\hline \multirow{3}{*}{\multicolumn{2}{|c|}{$\begin{array}{l}\text { cultivars treatment } \\
\text { interaction } \\
\text { L.S.D. at } 0.05\end{array}$}} & 4.95 & 4.01 & 5.83 & 5.10 & 4.87 & 8.76 \\
\hline & & 6.73 & 7.13 & 8.85 & 8.23 & 4.92 & 11.18 \\
\hline & & 8.96 & 8.20 & 10.18 & 9.13 & 7.95 & 14.90 \\
\hline
\end{tabular}


Table.7 Effect of Different Rates (0,100 and $200 \mathrm{Kg} / \mathrm{Fed})$ of Superphosphate on Some Growth Characters of Three Cultivars of Faba Bean Grown during Two Seasons under Field Conditions

\begin{tabular}{|c|c|c|c|c|c|c|c|}
\hline \multirow[t]{3}{*}{ Cultivars } & \multirow{3}{*}{$\begin{array}{l}\text { Super } \\
\text { phosphate } \\
(\mathrm{Kg} / \mathrm{fed})\end{array}$} & \multicolumn{6}{|c|}{ Crop parameters of faba bean plant } \\
\hline & & \multicolumn{3}{|c|}{$2012 / 2013$} & \multicolumn{3}{|c|}{$2013 / 2014$} \\
\hline & & $\begin{array}{l}\text { Plant height } \\
(\mathrm{cm})\end{array}$ & $\begin{array}{l}\text { Fresh weight } \\
\text { plant }(\mathrm{g})\end{array}$ & $\begin{array}{l}\text { Dry weight } \\
\text { plant }(\mathrm{g})\end{array}$ & $\begin{array}{l}\text { Plant height } \\
(\mathrm{cm})\end{array}$ & $\begin{array}{l}\text { Fresh weight } \\
\text { plant }(g)\end{array}$ & $\begin{array}{l}\text { Dry weight } \\
\text { plant }(\mathrm{g})\end{array}$ \\
\hline \multirow{4}{*}{$\begin{array}{l}\text { Giza3 } \\
\text { Mohassan }\end{array}$} & 0 & 29.13 & 9.88 & 0.91 & 28.12 & 9.00 & 0.86 \\
\hline & 100 & 32.55 & 10.00 & 0.94 & 33.18 & 10.35 & 0.89 \\
\hline & 200 & 33.00 & 10.13 & 0.95 & 35.15 & 11.00 & 0.91 \\
\hline & mean & 31.56 & 10.0 & 0.93 & 32.15 & 10.12 & 0.89 \\
\hline \multirow{4}{*}{ Giza 40} & 0 & 24.0 & 7.66 & 0.78 & 25.21 & 8.65 & 0.80 \\
\hline & 100 & 26.0 & 9.33 & 0.89 & 27.19 & 10.17 & 0.90 \\
\hline & 200 & 29.0 & 10.00 & 0.91 & 28.67 & 10.30 & 0.90 \\
\hline & mean & 26.33 & 8.99 & 0.86 & 27.02 & 9.71 & 0.87 \\
\hline \multirow{4}{*}{ Sakha1 } & 0 & 32.28 & 10.35 & 1.13 & 33.08 & 11.00 & 1.23 \\
\hline & 100 & 34.00 & 11.67 & 1.16 & 35.07 & 12.65 & 1.29 \\
\hline & 200 & 37.00 & 12.00 & 1.20 & 38.13 & 12.90 & 1.33 \\
\hline & mean & 34.43 & 11.34 & 1.16 & 35.43 & 12.18 & 1.28 \\
\hline \multirow{3}{*}{\multicolumn{2}{|c|}{$\begin{array}{l}\text { cultivars treatment } \\
\text { interaction } \\
\text { L.S.D. at } 0.05\end{array}$}} & 0.69 & 0.21 & 0.01 & 0.79 & 0.31 & 0.12 \\
\hline & & 0.98 & 0.13 & 0.12 & 1.10 & 0.29 & 0.09 \\
\hline & & 1.43 & 0.36 & 0.16 & 1.32 & 0.70 & 0.18 \\
\hline
\end{tabular}


Table.8 Effect of Superphosphate on Some Yield Component of Three Cultivars of Faba Bean under Field Conditions

\begin{tabular}{|c|c|c|c|c|c|c|c|c|c|c|c|}
\hline \multirow[t]{2}{*}{ Cultivars } & \multirow{2}{*}{$\begin{array}{l}\text { Superph- } \\
\text { osphate } \\
\text { Kg/fed }\end{array}$} & \multicolumn{5}{|c|}{$2012 / 2013$} & \multicolumn{5}{|c|}{$2013 / 2014$} \\
\hline & & $\begin{array}{l}\text { Plant } \\
\text { height } \\
(\mathrm{cm})\end{array}$ & $\begin{array}{l}\text { Branche } \\
\text { s/ plant }\end{array}$ & $\begin{array}{l}\text { No } \\
\text { seeds } \\
\text { pods }\end{array}$ & $\begin{array}{l}100 \\
\text { seed } \\
\text { weight } \\
(\mathrm{g})\end{array}$ & $\begin{array}{l}\text { Seed } \\
\text { weight } \\
\text { ardab/fe } \\
\text { d }\end{array}$ & $\begin{array}{l}\text { Plant } \\
\text { height } \\
(\mathrm{cm})\end{array}$ & $\begin{array}{l}\text { Branche } \\
\text { s/ plant }\end{array}$ & $\begin{array}{l}\text { No } \\
\text { seeds } \\
\text { pods }\end{array}$ & $\begin{array}{l}100 \\
\text { seed } \\
\text { weigh } \\
\mathrm{t}(\mathrm{g})\end{array}$ & $\begin{array}{l}\text { Seed } \\
\text { weight } \\
\text { ardab/fe } \\
\text { d }\end{array}$ \\
\hline \multirow{4}{*}{$\begin{array}{l}\text { Giza3 } \\
\text { Mohassan }\end{array}$} & 0 & 92.0 & 2.65 & 18.00 & 65 & 3.35 & 89 & 2.50 & 17 & 70 & 3.10 \\
\hline & 100 & 95.0 & 2.95 & 23.0 & 75 & 3.80 & 93 & 2.65 & 22 & 75 & 3.50 \\
\hline & 200 & 98.0 & 2.98 & 24.0 & 80 & 4.00 & 95 & 2.70 & 22 & 80 & 3.80 \\
\hline & Mean & 95 & 2.86 & 21.67 & 73.33 & 3.72 & 92.33 & 2.62 & $\begin{array}{c}20.3 \\
3\end{array}$ & 75 & 3.47 \\
\hline \multirow{4}{*}{ Giza 40} & 0 & 85 & 2.00 & 13 & 65 & 3.13 & 83 & 2.00 & 11 & 60 & 3.00 \\
\hline & 100 & 89 & 2.20 & 16 & 76 & 3.20 & 88 & 2.23 & 15 & 65 & 3.25 \\
\hline & 200 & 90 & 2.33 & 17 & 70 & 3.17 & 89 & 2.33 & 16 & 70 & 3.35 \\
\hline & Mean & 88 & 2.18 & 15.33 & 68.33 & 3.27 & 86.67 & 2.19 & 14 & 65 & 3.20 \\
\hline \multirow{4}{*}{ Sakha1 } & 0 & 95 & 2.55 & 19 & 80 & 3.90 & 93 & 2.60 & 20 & 85 & 3.70 \\
\hline & 100 & 100 & 2.60 & 26 & 90 & 4.25 & 99 & 2.70 & 26 & 90 & 4.00 \\
\hline & 200 & 103 & 2.65 & 27 & 95 & 4.40 & 101 & 2.70 & 28 & 95 & 4.50 \\
\hline & Mean & 99.33 & 2.60 & 24 & 88.33 & 4.18 & 97.67 & 2.67 & $\begin{array}{c}24.6 \\
7 \\
\end{array}$ & 90 & 4.07 \\
\hline \multirow{3}{*}{\multicolumn{2}{|c|}{ L.S.D. at 0.05}} & 1.50 & 0.15 & 0.54 & 0.40 & 0.66 & 1.27 & 0.11 & 0.61 & 8.35 & 0.11 \\
\hline & & 3.04 & 0.31 & 1.31 & 0.98 & 1.00 & 2.13 & 0.34 & 1.14 & 1.42 & 0.31 \\
\hline & & 3.55 & 0.63 & 1.59 & 1.35 & 1.60 & 3.80 & 0.65 & 1.68 & 1.32 & 0.91 \\
\hline
\end{tabular}


Table.9 Effect of Superphosphate and Intercropping Three Cultivars of Faba Bean with Wheat Treatments on Chemical Composition of Three Cultivars of Faba Bean under Field Conditions

\begin{tabular}{|l|c|c|c|c|c|c|c|}
\hline Cultivars & Treatments & \multicolumn{3}{|c|}{$2012 / 2013$} & \multicolumn{3}{c|}{$2013 / 2014$} \\
\cline { 2 - 7 } & & $\mathrm{P} \%$ & $\mathrm{~N} \%$ & Protein \% & $\mathrm{P} \%$ & $\mathrm{~N} \%$ & Protein \% \\
\hline Giza3 & 0 & 0.40 & 4.15 & 25.94 & 0.37 & 4.10 & 25.63 \\
Mohassan & 100 & 0.50 & 4.50 & 28.13 & 0.47 & 4.40 & 27.50 \\
& 200 & 0.57 & 4.80 & 30.0 & 0.54 & 4.75 & 29.69 \\
\hline Giza 40 & 0 & 0.33 & 3.99 & 24.94 & 0.32 & 3.88 & 24.25 \\
& 100 & 0.40 & 4.15 & 25.94 & 0.38 & 4.12 & 25.75 \\
& 200 & 0.45 & 4.30 & 26.88 & 0.43 & 4.25 & 26.56 \\
\hline \multirow{4}{*}{ Sakha1 } & 0 & 0.44 & 4.66 & 29.13 & 0.41 & 4.63 & 28.94 \\
& 100 & 0.52 & 4.90 & 30.63 & 0.49 & 4.85 & 30.31 \\
& 200 & 0.59 & 5.00 & 31.25 & 0.55 & 4.90 & 30.63 \\
\hline Giza3 & 2 row X 2 row (wheat) & 0.36 & 4.00 & 25.63 & 0.33 & 4.00 & 25.00 \\
& 3 row X 3 row (wheat) & 0.45 & 4.40 & 27.50 & 0.44 & 4.35 & 27.19 \\
& 2 row X 4 row (wheat) & 0.53 & 4.60 & 28.75 & 0.50 & 4.55 & 28.44 \\
& Faba bean alone & 0.35 & 4.00 & 25.00 & 0.32 & 3.95 & 24.69 \\
\hline Giza 40 & 2 row X 2 row (wheat) & 0.32 & 3.91 & 24.43 & 0.31 & 3.77 & 23.56 \\
& 3 row X 3 row (wheat) & 0.36 & 4.00 & 25.00 & 0.32 & 3.98 & 24.88 \\
& 2 row X 4 row (wheat) & 0.40 & 4.10 & 25.63 & 0.36 & 4.00 & 25.00 \\
& Faba bean alone & 0.30 & 4.88 & 24.25 & 0.30 & 3.70 & 23.13 \\
\hline \multirow{3}{*}{ Sakha1 } & 2 row X 2 row (wheat) & 0.42 & 4.13 & 25.81 & 0.38 & 4.20 & 26.25 \\
& 3 row X 3 row (wheat) & 0.46 & 4.50 & 28.13 & 0.44 & 4.45 & 27.81 \\
& 2 row X 4 row (wheat) & 0.55 & 4.70 & 29.38 & 0.53 & 4.68 & 29.25 \\
& Faba bean alone & 0.40 & 4.00 & 25.0 & 0.36 & 4.10 & 25.63 \\
\hline
\end{tabular}

The present results in table (9) are agreed with those obtained by Mengle and Krikby (1987). They found that, in seeds and grains, $\mathrm{P}$ contents in the range of 0.4 to 0.5 $\%$ in the dry matter.

Faba bean (Viciafaba L.) is one of the most important legume crops. It is infested with many fungal pathogens causing considerable yield losses where dampingoff, root-rot, wilted diseases affecting faba bean production in Egypt Abdel-Kader et al., (2011). In this study induced resistance against root-rot were shown when intercropping and fertilization also decreased the fungal growth of $R$. soloni in vivo. The greenhouse results indicate that such intercropping reduce the percentage of pre-emergence and post-emergence damping-off. This results are agreement with the finding of (Boudreau and Mundt 1992, and Fininsa 1996) and (Kinane and
Lyngkjaer 2002). It seems that intercropping affected positively to somewhat faba plant height. Meanwhile, the results revealed that intercropping three cultivars of faba bean (Giza 3 Mohassan, Giza 40 and sakha 1) with wheat sakha 93 increased significantly plant height after harvest, number of branches, plant number of pods/plant, 100 seed weight/g and seed weight ardab/fed in both seasons of the three cultivar of faba bean.

In green house and field condition, super phosphate treatment significantly reduced pre and post-emergence damping off as well as root-rot disease, consequently increased germination percentage and healthy plants. In addition, super phosphate treatment increased significantly vegetation growth parameters and yield component. Such enhancement 
effect of super phosphate on the vegetative growth parameters might be attributed to its effect on nodulation and yield parameters ( Jain et al., 1999, Khurana and Sharma 2000, Rudresh et al., 2005 and Rania et al., 2010). Application of phosphorus fertilizer increased plant height harvest, number of branches / plant. Number of pods/plant, 100-seed weight/g) and seed weight ardab/fed in both seasons of the three cultivars of faba bean. These results are in agreement with Ahmed et al., (1992),they found the, the application phosphorus fertilizer increased significantly protein content and carbohydrate content in seed and different plant parts.

\section{References}

AOAC (Association of Official Analytical Chemists) (1980) Official methods of analysis of the Association of Official Analytical Chemists. 13th ed. Washington, DC.

Abdel-Kader M.M., El-Maugy N.S., and Lashin S.M., (2011) Essential oils and trichodermaharzianum as an integrated control measure against faba bean root-rot pathogens. Journal Plant Protection Research 51:306-313

Ahmed S. A., Abdel-Mottaleb S. A., Ismail S. A., and El-Gendi F.H.(1992). Response of broad bean (Vicia Raba L.) to application of phosphorus of phosphorus and zinic. Egyptian Journal of Applied Sciences 7,11:418426

Andrews D.J., and Kassam A.H., (1976) The important of multiple cropping in increasing world food supplies .In:papendick R.I., Sanchez A., Triplett G. B.(eds): Multiple cropping. As a special publication 27. American Society of Agronomy, Madison 1-10

Boudreau M.A.,and Mundt C.C. (1992) Mechanisms of alterations in bean rust epidemiology due to intercropping with maize. Phytopathology 82:1051-1060

Chapman H.D. and Pratt P.P., (1961) Methods of analysis for soils plants and waters PP. 201-203. University of California, Division of Agricultural science, Barkely, USA.

Chavan J. K., Kute L.S., and Kadam S.S.,(1989) Soybean In: Salunkhe DD, Kadam SS, editors. CRC handbook of world food legumes. Boca Raton: CRC Press; 1989. pp. 223-245.

El-helaly A. F., Elarosi H. M., Assawah M.W. and Abol-Wafa M. T., (1970) Studies on damping-off and root-rots of bean in UAR (Egypt). Egypt. J. Phytopathol., 2: $41-57$.

Fininsa, C. (1996) Effect of bean and maize intercropping on bean common bacterial blight and rust diseases. International Journal Pest Management, 42: 51-54.

Gomez, K.A. and Gomez, A. A. (1984) Statistical Procedures for Agricultural Research pp. 424-452. John wily and Sons. Inc. New York

Jafar Nakhost Majid Ashoori (2014) Effect of biologic fertilization, mineral phosphorus and nitrogen on faba bean yield and yield components in northern Iran. Indian Journal of fundamental and applied Life Science.(4):3 2231-6345

Jain, P. C., Kushawaha P. S., Dhakal U. S., Khan H. and Trivedi S. M., (1999). Response of chickpea (Cicer arietinum L.) to phosphorus and biofertilizer. Legume Res 22: 241244.

Khurana, A. S., and Sharma, P. (2000) Effect of dual inoculation of phosphate solubilizing bacteria, Bradyrhizobium sp. (Cicer) and phosphorus on nitrogen fixation on yield of chickpea. Indian journal Pulses Research 13: 66-67. 
Kinane, J. Lyngkjaer M. F. (2002) Effect of barley-legume intercrop on disease frequency in on organic farming system 6. Conference of European Foundation for Plant Pathology (EFFP 2002), Prague (CZ) 8-14 Sep 2002. Plant Protection Science. 38: 227-231

Kontturi, M., Laine A., Niskanen M., Hurme T., Hyovela M., and Peltonen-Sainio P. (2011) Pea-oat intercrops to sustain lodging resistance and yield formation in northern European conditions. Acta Agriculturae Scandinavica, Section B-Soil \& Plant Science. 61:612-621

Metwaly M.M.M. (2004) Resistance induction against diseases of faba bean crop .Ph D. Thesis plant pathology Dept. Fac. Agric. Suez Canal Univ. Egypt.2231- 6345

Negm, M. A., Abdel-Reham M. A., and Montasser S. Y. (1992)Effect of different rates and frequencies of foliar applications of phosphorus on sunflower growing on calcareous soils. Egypt journal soil Science. 32:513-512

Omar S. A. (1986) Pathological studies on root-rot disease of faba bean (Viciefaba L.) 11 Int. Conference Society and Dem. Res. Cairo, Egypt, PP 33-48

Rania Z. El-shennawy, Omran M. M. and Abd El-Motteleb (2010) Effect of phosphorus fertilizer treatments on incidence of Fusarium root-rot/ wilt disease complex, and yield components of lupine, chick pea and lentil crops. Arab university Journals Agricultural Science Ain Shams University, Cairo 18(1) 193-202

Rudresh D. L., Shivaprokash M. K. and Prasad R.D.,(2005) Effect of combined application of Rhizobium, phosphate solubilizing bacterium and Trichoderma spp. On growth, nutrient uptake and yield chicpea (Cicer arietinum L.). Applied Soil Ecology 28:139-146

Singh, K. N., Prased R. D. and Tomar V. P. S. (1981) Response of French been to different levels of nitrogen and phosphorus in Nil Giri-Hills under rainfed conditions. Indian Journal of Agronomy 26:101-102

Soleman N.K., Mikhail M. S., HarbP.k. and Khalil E. M. (1988) Response of bread bean plants infected with Rhizoctonia solani of growth regulators and calcium. Egypt Journal Phytopathology. 20(1): 1-11

Tripathi R. D., Srivastava G. P., Misra M. S. and Pandey S. C. (1971) Protein content in some varieties of legume, The Allah Abad Farmer. 16:291- 294

Vandermeer J., Van-NoordwiJk M. Anderson J., Ong C., and Perfecto I.,(1998) Global change and multispecies agroecosystems : concepts and issues. Agriculture, Ecosystems Environment 67: 1-22.

\section{How to cite this article:}

Abeer M. Mousa and Sahar A. El-Sayed. 2016. Effect of Intercropping and Phosphorus Fertilizer Treatments on Incidence of Rhizoctonia Root-Rot Disease of Faba Bean. Int.J.Curr.Microbiol.App.Sci.5(4): 850-863. doi: http://dx.doi.org/10.20546/ijcmas.2016.504.097 